\title{
Chronic idiopathic axonal polyneuropathy: a five year follow up
}

\author{
N C Notermans, J H J Wokke, Y van der Graaf, H Franssen, G W van Dijk, \\ F G I Jennekens
}

\begin{abstract}
Seventy five patients with chronic idiopathic axonal polyneuropathy (CIAP) were studied for five years. The standardised and quantified neurological examination shows that progression of CIAP is slow, and handicap, if present, is not severe. During the follow up period a definite cause of the neuropathy was found in only four patients (two hereditary motor and sensory neuropathy type 2 , one sensory chronic inflammatory demyelinating polyneuropathy, one alcoholic neuropathy). At the end of the follow up CIAP was not related to malignancy or gammopathy. Routine repetition of laboratory tests was not informative and these tests should be performed on clinical grounds only.
\end{abstract}

$(\mathcal{F}$ Neurol Neurosurg Psychiatry 1994;57:1525-1527)

In $10-15 \%$ of patients with chronic polyneuropathy a cause cannot be detected despite thorough evaluation. ${ }^{1}$ In an earlier study we have described a group of 75 patients with a chronic idiopathic axonal polyneuropathy (CIAP). ${ }^{2}$ Briefly, these idiopathic polyneuropathies are sensorimotor or sensory in nature, and electrophysiological and pathological studies are compatible with axonal degeneration. ${ }^{2}$

To determine whether an aetiological cause could eventually be found and to monitor the clinical progression of the disease, we prospectively followed up the same group of 75 patients for a mean period of five years.

\section{Materials and methods}

Seventy five patients with CIAP entered the study between 1987 and 1991. The clinical features of this group have been presented elsewhere. ${ }^{2}$ The mean age at the onset of symptoms was 56.5 (range 45-70) years. On clinical grounds the neuropathy was sensorimotor in 44 patients ( 21 men), sensory in 29 patients (23 men), and purely motor in two men. Electrophysiological studies at entry into the study and nerve biopsy studies identified axonal degeneration. Tests for anti- bodies against GM1 ganglioside, myelin associated glycoprotein, and sulphatides were negative. Possible aetiological factors had been thoroughly evaluated and found negative at the start of the study. ${ }^{2}$

During the five years of follow up the patient evaluation was done by the same neurologist $(\mathrm{NCN})$. Medical and family histories were taken each year. ${ }^{34}$ The following tests were performed twice a year: (a) measurement of strength (MRC grading system) in six muscles of each arm (deltoid, biceps and triceps brachii, finger extensors, finger flexors, and first dorsal interosseus) and six muscles of each leg (iliopsoas, quadriceps femoris, hamstrings, anterior tibial, gastrocnemius, and peroneal). Summation of test results could lead to a maximum score of 120 ; (b) quantification of sensory functions. The sensory system was graded as follows: both touch and pin prick sense: normal $=4$, distal to wrist/ankle abnormal $=3$, distal half fore$\mathrm{arm} / \mathrm{leg}$ abnormal $=2$, distal to elbow $/$ knee abnormal $=1$, distal to axilla/groin abnormal $=0$; vibration sense: tuning fork perception $(128 \mathrm{~Hz})$ on middle finger/hallux $=4$, ulnar styloid/medial malleolus $=3$, elbow $/$ knee $=2$, clavicle/iliac crest $=1$, no perception $=0$; joint position sense of middle finger/hallux: normal $=2$, diminished $=1$, absent $=0$. Summation of all sensory modalities could lead to a maximum score of 56 . Disability was determined with the modified Rankin disability scale. ${ }^{5}$

Once every year haemoglobin, packed cell volume, leucocytes, platelets, erythrocyte sedimentation rate, serum glucose, renal function, electrolytes, and liver enzymes were measured. At the beginning and end of the study serum agar gel and immunoelectrophoresis and analysis of antinuclear antibodies were carried out and chest radiographs were taken.

For the statistical analysis the results of sum scores can be considered as an ordinal scale, and are presented in percentiles. The non-parametric Wilcoxon rank sum test was used to test differences between the start and the end of the study for the whole group and for subgroups of patients with sensorimotor or sensory neuropathy. Values of $p<0.05$ were considered to be significant. 
Mean sensory and motor sum scores of patients with clinically sensory and sensorimotor neuropathies (50 percentiles)

\begin{tabular}{|c|c|c|c|}
\hline & $\begin{array}{l}A l l \\
(n=71)\end{array}$ & $\begin{array}{l}\text { Sensorimotor } \\
(n=50)\end{array}$ & $\begin{array}{l}\text { Sensory } \\
(n=21)\end{array}$ \\
\hline \multicolumn{4}{|c|}{ Sensory score ${ }^{\star}$ : } \\
\hline Start & 44 & 44 & 44 \\
\hline 1.5 years & 44 & 44 & 42 \\
\hline 3 years & 42 & 42 & 42 \\
\hline 5 years & 42 & 42 & 42 \\
\hline \multicolumn{4}{|c|}{ Motor scoret: } \\
\hline Start & 118 & 116 & 120 \\
\hline 1.5 years & 118 & 116 & 120 \\
\hline 3 years & 117 & 116 & 120 \\
\hline 5 years & 116 & 116 & 120 \\
\hline
\end{tabular}

$\star$ Maximum sensory sum score $=56 ;$ tmaximum motor sum score $=120$.

\section{Results}

Retrospectively, the onset of the neuropathy was 6.3 (SD 4.3 ) years before the study started. The mean duration of the prospective period was 4.7 (SD 0.9) years.

Follow up disclosed a cause of the neuropathy in only four of the 75 patients (5\%). In two of them, aged 63 and 65 at the start of the study, a hereditary cause seemed likely as a sensorimotor axonal neuropathy presented in sibs at the age of 51 and 56 years. Probably these patients had a hereditary motor and sensory neuropathy (HMSN) type 2. The family history of all the other patients (including their 156 children) was negative. One man with a sensory neuropathy had a slightly raised CSF protein content of $0.55 \mathrm{~g} / 1$ (95\% confidence interval $0 \cdot 15-0 \cdot 45$ ). On entry the electrophysiological examination was compatible with axonal degeneration. After a follow up period of two years, in which no progression occurred, the neuropathy relapsed after an attack of influenza. As features of demyelination were now present on electrophysiological examination (in four motor nerves temporal dispersion of at least $30 \%$ of the compound muscle action potential between stimulation sites in the lower arm or lower leg, reduction of conduction velocity of more than $70 \%$ of the normal limit; in six sensory nerves absent or reduced sensory nerve action potentials), the diagnosis was changed to chronic inflammatory demyelinating polyneuropathy. In the fourth patient, alcohol abuse became apparent. These four patients have been excluded from further discussion.

During the prospective follow up period the polyneuropathy remained sensorimotor in all patients with a sensorimotor neuropathy ( $n=42$ ).

In seven of 28 patients with clinical features of a sensory neuropathy on entry, the neuropathy became sensorimotor. In five of these seven patients, the electrophysiological evaluation had already shown subclinical motor abnormalities (decreased amplitude of the compound motor action potential, presence of fibrillations or positive waves). In 13 of the other 21 patients, in whom the neuropathy remained sensory during follow up, subclinical motor electrophysiological abnormalities were also found.

One patient had a pure motor neuropathy on clinical grounds, whereas electrophysiological examination showed subclinical sensory abnormalities. During follow up the neuropathy became clinically sensorimotor.

The clinical course of the neuropathy in all patients $(n=71)$ between entry and end of the study, as determined by the sum scores, was progressive (Wilcoxon rank sum test, $p<$ $0 \cdot 001)$. No significant differences were found between the sensorimotor and sensory neuropathy (Wilcoxon rank sum test, $\mathrm{p}>0.05$; table). At the start of the study, the score on the modified Rankin scale was 1 point in 35 patients, 2 points in 35 other patients, and 3 points in one patient. After the follow up 1 point was scored in five patients (all with a sensory neuropathy) and 2 points in 63 . Three patients became confined to a wheelchair and scored 3 points, which was largely due to other current illnesses (hemiparesis caused by a lacunar infarction, arthrosis of the knees, or replacement of a hip joint). Twenty three of 50 patients with a sensorimotor neuropathy needed a walking cane and 20 patients also needed ankle braces. This is in contrast with the finding that only one of the 21 patients with a sensory neuropathy needed a walking cane.

No metabolic or toxic causes of the neuropathy were found during follow up. None of the patients developed a gammopathy. A malignancy was detected in four patients with a sensorimotor neuropathy (two squamous cell carcinomas of the lung, one colon carcinoma, and one carcinoma of the eye socket). Two of the four patients died as a result of the carcinoma after two years. The clinical course of the neuropathy and the neurophysiological investigations of these four patients did not change before, during, and after the treatment of the carcinoma. The neuropathy of these patients showed no rapid progression.

\section{Discussion}

This study shows that during a prospective follow up period of 4.7 years, CIAP was slowly progressive. The clinical course before patients entered the study had two patterns: in sensorimotor neuropathy the course was slowly progressive and in sensory neuropathy the clinical course more often had a tendency to stabilise. ${ }^{2}$ During the prospective follow up period, we did not find this difference in clinical course on the basis of the sum scores and Rankin scale. The only difference between the sensorimotor and sensory neuropathy was that patients with a sensory neuropathy used less devices. Another finding of this study is that CIAP is mostly a sensorimotor neuropathy if clinical and electrophysiological criteria are considered (63 of 71 patients; $89 \%$ ).

At the start of the study we hypothesised that some patients may have a gammopathy or a malignancy as a cause for CIAP. ${ }^{6}$ In none of our patients was there evidence for $\mathrm{M}$-protein synthesis. Only $1 \%$ of our patients developed a carcinoma. This incidence is the same as in the normal population corrected 
for age. ${ }^{8}$ Therefore, malignancy is an unlikely explanation for CIAP. Also, the clinical course of CIAP does not parallel that of a paramalignant microvasculitis. ${ }^{9}$

A cause for the neuropathy could only be found in four patients. During the follow up period the suspicion of a specific cause for the neuropathy was based on clinical grounds and repeated family history, and not on the routinely repeated laboratory investigations. ${ }^{3}$ Ancillary investigations, such as repeated lumbar puncture or electrophysiological examination performed on indication of a change in the clinical course of the polyneuropathy, may be helpful. The patient with a sensory polyneuropathy and a sudden relapse may have a sensory inflammatory demyelinating polyneuropathy..$^{10}$

In the study of McLeod et al, ${ }^{1} 47$ of 67 patients retrospectively identified with an idiopathic neuropathy were reviewed for a second time after a median period of three years. The main conclusions of both studies are similar. They determined a possible aetiological factor in $36 \%$ (17 of 47); six of these 17 patients developed a malignancy, and interestingly three had a gammopathy, which was not found in our series. We detected a cause for the neuropathy after long term follow up in only $5 \%$ (four of 75 patients). The different percentages may be explained by our study being prospective, with a standardised protocol.

In conclusion, even though the aetiology of CIAP has not been elucidated yet, CIAP shows an overall benign course with a good long term prognosis. After an initial extensive search for the cause of the polyneuropathy, yearly repetition of routine laboratory investigations does not provide any additional information on the aetiology and these tests should therefore be performed on clinical grounds only if suspicion of an intercurrent illness arises.

We thank Dr W H J P Linssen and W P Vandertop for their critical review of the manuscript.

1 McLeod JG, Tuck RR, Pollard JD, Cameron J, Walsh JC. Chronic polyneuropathy of undetermined cause. ₹ Neurol Neurosurg Psychiatry 1984;47:530-5.

2 Notermans NC, Wokke JHJ, Franssen H, et al. Chronic idiopathic polyneuropathy presenting in middle or old age: a clinical and electrophysiological study of 75 patients. $f$ Neurol Neurosurg Psychiatry 1993;56: 1066-71.

3 Dyck PJ, Oviatt KF, Lambert EH. Intensive evaluation of referred neuropathies yields improved diagnosis. Ann Neurol 1981;10:222-6.

4 Harding A, Thomas PK. The clinical features of hereditary motor and sensory neuropathies type 1 and 2 . Brain 1980;103:259-80.

5 Van Swieten JC, Koudstaal PJ, Visser MC, et al. Interobserver agreement for assessment of handicap in stroke patients. Stroke 1988;19:604-7.

6 Julien J, Vital C, Vallat JM, et al. Chronic demyelinating neuropathy with IgM-producing lymphocytes in peripheral nerve and delayed appearance of 'benign' monoclonal gammopathy. Neurology 1984;34:1387-9.

7 Horwich MS, Cho L, Porro RS, Posner JB. Subacute sensory neuropathy: a remote effect of cancer. Ann Neurol 1977;20:535-7.

8 Visser O, Coebergh JWW, Schouten LJ. Incidence of cancer in the Netherlands. Second report of the Netherlands cancer registration 1990 .

9 Oh SI, Slaughter R, Harrel L. Paraneoplastic vasculitic neuropathy: a treatable neuropathy. Muscle Nerve 1991;14:152-6.

10 Oh SJ, Joy JL, Kuruoglu R. Chronic sensory demyelinating neuropathy: chronic inflammatory demyelinating polyneuropathy presenting as a pure sensory neuropathy. $\mathcal{F}$ Neurol Neurosurg Psychiatry 1992;55:677-80. 